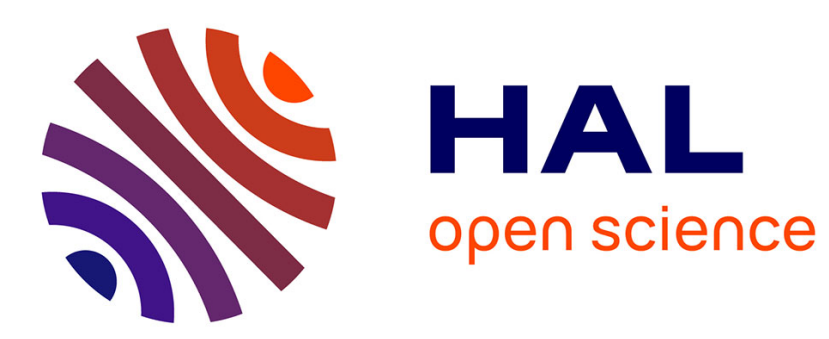

\title{
Multicast Channel Communication with Interactive Receiver Cooperation over Orthogonal Links
}

\author{
Victor Exposito, Sheng Yang, Nicolas Gresset
}

\section{To cite this version:}

Victor Exposito, Sheng Yang, Nicolas Gresset. Multicast Channel Communication with Interactive Receiver Cooperation over Orthogonal Links. IEEE International Conference on Communications (ICC 2017), IEEE, May 2017, Paris, France. 10.1109/ICC.2017.7996565 . hal-01504352

\section{HAL Id: hal-01504352 https://hal.science/hal-01504352}

Submitted on 10 Jun 2020

HAL is a multi-disciplinary open access archive for the deposit and dissemination of scientific research documents, whether they are published or not. The documents may come from teaching and research institutions in France or abroad, or from public or private research centers.
L'archive ouverte pluridisciplinaire HAL, est destinée au dépôt et à la diffusion de documents scientifiques de niveau recherche, publiés ou non, émanant des établissements d'enseignement et de recherche français ou étrangers, des laboratoires publics ou privés. 


\title{
Multicast Channel Communication with Interactive Receiver Cooperation over Orthogonal Links
}

\author{
Victor Exposito*†, Sheng Yang*, Nicolas Gresset ${ }^{\dagger}$ \\ *L2S (UMR CNRS 8506), CentraleSupélec, Université Paris-Sud, Université Paris-Saclay, Gif-sur-Yvette, France \\ ${ }^{\dagger}$ Mitsubishi Electric R\&D Centre Europe, Rennes, France \\ Email: \{victor.exposito,sheng.yang\}@ centralesupelec.fr, \{v.exposito,n.gresset\}@ fr.merce.mee.com
}

\begin{abstract}
We consider the multicast channel (MC), in which a transmitter broadcasts only a common message to two receivers. In our previous work, we have derived information-theoretic upper and lower bounds and showed that the two-round receiver cooperation (2RC) scheme, in which the receivers interactively perform compress-forward and then decode-forward, improves the MC achievable rate over different schemes from the literature, in the general case of full-duplex bi-directional nonorthogonal cooperation links. In this paper, we concentrate on the orthogonal cooperation to identify the properties of this interactive cooperation. We show that the full-duplexing between the cooperation links is not essential to take advantage of most of the gain offered by the $2 \mathrm{RC}$ full-duplex scheme, while the full-duplexing between the MC and the cooperation links brings most of the gain.
\end{abstract}

\section{INTRODUCTION}

The increase of multimedia content delivery in wireless communication systems is leading, among many, to a spectrum crunch or interference intensification. In traditional broadcasting systems the same content is intended to a group of users. Multilayer strategies can be used so that the transmission rate is not limited by the weakest user in terms of channel quality. However, if all users wish to obtain the same content quality, the weakest user would set the rate and thus impact the whole group. With the recent study of device-to-device (D2D) mechanisms in standards, user cooperation in close proximity becomes possible and would benefit all the users. While the full-duplex is an interesting way to improve the capacity of the system, in state-of-the-art hardware it remains challenging.

In this work, we investigate the broadcast channel (BC) with one transmitter sending a common message to two receivers, also called a multicast channel (MC) in this case. The receivers can cooperate through a cooperation link. Since the receivers also transmit signals through the cooperation link, our channel is a mixture of the $\mathrm{MC}$ and the relay channel ( $\mathrm{RC}) . \mathrm{BCs}$ were introduced in [1]. The capacity of BCs remains unknown except for special cases such as the stochastically degraded BC [2] for which superposition coding is optimal. The largest achievable rate-region known to date for the general case is based on binning [3]. RCs were introduced in [4]. The fundamental relaying strategies called compress-forward (CF) and decode-forward (DF) were proposed in [5]. Relaying strategies based on $\mathrm{CF}$ and $\mathrm{DF}$ were proposed for larger networks [6]-[10]. The capacity of the BC with cooperation is unknown in general, except for special cases such as the physically degraded main channel.

The setup of the current work has been partially studied in [11], referred to as BCs with cooperative decoders, and in [12], [13], referred to as relay BCs. A BC with orthogonal cooperation links was considered in [11]. In [12], [13], although the cooperation links are not restricted to be orthogonal, the authors assumed that either the main channel is degraded or the cooperation link is uni-directional. It is worth noting that achievable rate regions of both common and private messages were provided in [11]-[13]. In [14], the authors extended the results of [11]-[13] for the general MC with receiver cooperation over full-duplex bi-directional non-orthogonal cooperation links, and presented a two-round interactive receiver cooperation (2RC) scheme for which they characterized the benefit of a cooperation using both $\mathrm{CF}$ and DF in terms of achievable multicast rate through an information-theoretic analysis. The $2 \mathrm{RC}$ scheme is based on block Markov superposition coding - as [7]-[9], but contrary to [11]-[13] - to provide the general result where the two receivers are allowed to cooperate with each other over fullduplex bi-directional non-orthogonal cooperation links, and uses short messages - as [9], but contrary to [7], [8], [11]-[13] - to permit the use of DF. While a study of the non-orthogonal case would lead to a more general result, the non-linearity of the bounds in this latter case would not be didactic and in some cases would not lead to a closed-form expression. Thus, in order to identify the properties of this interactive cooperation which enhances the achievable rate of the MC with receiver cooperation, we break down the scheme into orthogonal cases. The comparison of those cases helps us understand the behavior of the system and underline rules on how such an interactive cooperation should be apprehended.

The remainder of the paper is organized as follows. Sec. II introduces the system model, as well as the orthogonal case and the Single-Input Single-Output (SISO) Gaussian MC case. We briefly recall the performances of the $2 \mathrm{RC}$ scheme compared to other non-interactive schemes. Sec. III presents the analytical study of the 2RC scheme in the cases of 1) full-duplex cooperation, 2) resource-sharing cooperation, and 3) half-duplex cooperation. Numerical results are provided in Sec. IV for the SISO Gaussian MC counterpart of all those cases to better understand the role this interactive cooperation plays in this system. 


\section{SYSTEM MODEL}

We consider a simple multicast network where one transmitter sends the same information to two receivers through the main channel. The two receivers can cooperate with each other in full-duplex, i.e., they can transmit and receive simultaneously, through a bi-directional non-orthogonal cooperation link. This setup includes, 1) the cooperation links orthogonal to the main channel, orthogonal links being either physically separated medium, e.g., using different transmission technologies over different resources, or created with artificial orthogonalization, e.g., in time or frequency and, 2) the halfduplex mode if the receivers transmit and receive at a different time. To derive upper and lower bounds on the achievable rate, we resort to information theory. The current channel belongs to a class of stationary memoryless channels shown in Fig. $2,\left(\mathcal{X} \times \mathcal{X}_{1} \times \mathcal{X}_{2}, p\left(y_{1}, y_{2} \mid x, x_{1}, x_{2}\right), \mathcal{Y}_{1} \times \mathcal{Y}_{2}\right)$, defined as $p\left(y_{1}^{n}, y_{2}^{n} \mid x^{n}, x_{1}^{n}, x_{2}^{n}\right)=\prod_{i=1}^{n} p\left(y_{1 i}, y_{2 i} \mid x_{i}, x_{1 i}, x_{2 i}\right)$ where $x^{n} \in \mathcal{X}^{n}, x_{1}^{n} \in \mathcal{X}_{1}^{n}$, and $x_{2}^{n} \in \mathcal{X}_{2}^{n}$ are the sequences of transmitted signals from the transmitter, receiver 1 and 2 , respectively, and $y_{1}^{n} \in \mathcal{Y}_{1}^{n}$ and $y_{2}^{n} \in \mathcal{Y}_{2}^{n}$ are the sequences of received signals at the receivers 1 and 2, respectively. The probability distribution of the channel is known at every node (perfect channel state information (CSI) at the transmitter and receivers) by assumption. The common message $M$ is assumed to be uniformly distributed in $\mathcal{M} \triangleq\left[1: 2^{n R}\right]$ where $R$ is the number of bits per channel use. An encoder at the transmitter side is a map $f_{i}^{(n)}$ from the message $M$ to the sequence of input symbols $x^{n}$, an encoder at the receiver $k, k=1,2$, is a sequence of maps $\left\{f_{k, i}^{(n)}\right\}_{i}$ from the past received symbols $y_{k}^{i-1}$ to the transmitted symbol $x_{k, i}$. A decoder at the receiver $k$ is a map $\left\{g_{k, i}^{(n)}\right\}_{i}$ from the received sequence $y_{k}^{n}$ to $\hat{M}^{(k)} \in \mathcal{M}$. The probability of error is defined as $P_{e}^{(n)} \triangleq \operatorname{Pr}\left(M \neq \hat{M}^{\prime}\right.$ or $\left.M \neq \hat{M}^{\prime \prime}\right)$. Finally, a rate $R$ is achievable if there exist a sequence of encoders/decoders $\left(f_{i}^{(n)},\left\{f_{1, i}^{(n)}\right\}_{i},\left\{f_{2, i}^{(n)}\right\}_{i},\left\{g_{1, i}^{(n)}\right\}_{i},\left\{g_{2, i}^{(n)}\right\}_{i}\right)$ such that $P_{e}^{(n)} \rightarrow 0$ when $n \rightarrow \infty$.

In a previous work [14], we have shown that the $2 \mathrm{RC}$ scheme outperforms 1) the non-interactive CF scheme which turned out to achieve the same rate as the the noisy network coding (NNC) scheme [7], [8], and the short message noisy network coding (SNNC) scheme [9] (since the achievable rate of the SNNC with backward decoding or sliding window decoding is equal to the one of the NNC with joint decoding), and 2) the non-interactive DF scheme which turned out to achieve the same rate as the SNNC with a DF option (SNNCDF) scheme [9] when forced to use the DF option to ensure the multicasting property. We sum up this previous work in Fig. 1, where we represent the cutset upper bound and the four lower bounds presented in the aforementioned paper which are the $2 \mathrm{RC}$, the non-interactive $\mathrm{CF}$ and DF schemes, and the "no cooperation" scheme - and compare them in the non-orthogonal case. Proofs are omitted and the readers are referred to [7]-[9], [14]-[16] for details. Fig. 1 represents, for a rate $R$ that is expected to be achieved and a fixed

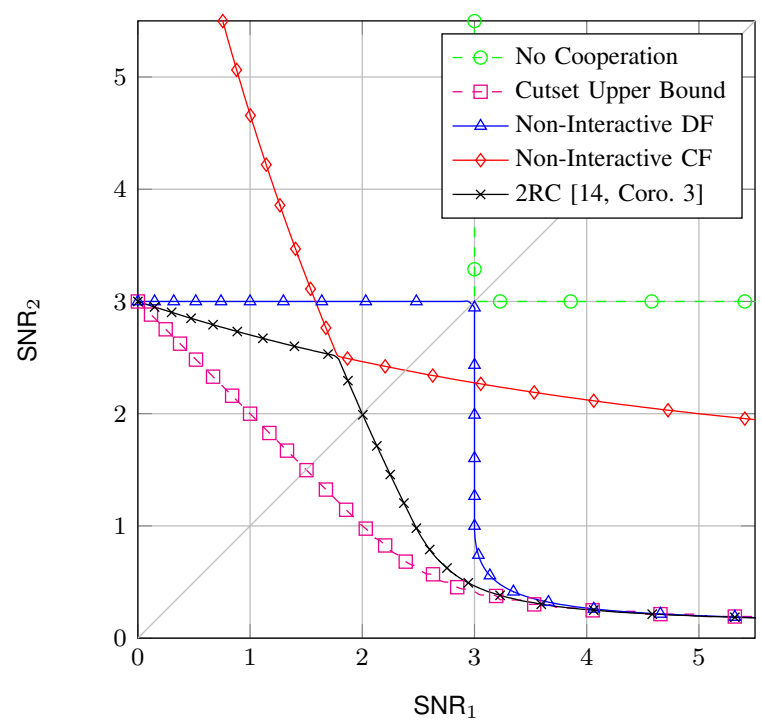

Fig. 1: Comparison of the upper and lower bounds on the values of the main channel gains for the Gaussian MC with receiver cooperation supporting a given rate $R=2 \mathrm{bit} / \mathrm{s} / \mathrm{Hz}$ for a cooperation of $\left(\mathrm{SNR}_{12}=2, \mathrm{SNR}_{21}=5\right)$. In order to distinguish well the curves the SNRs are linear and not in $\mathrm{dB}$.

given level of cooperation $\left(\mathrm{SNR}_{12}, \mathrm{SNR}_{21}\right)$, all the values of the main channel gains $\left(\mathrm{SNR}_{1}, \mathrm{SNR}_{2}\right)$ that allow the receivers to be able to decode with the different schemes presented above as well as the cutset upper bound. For a given scheme, a couple $\left(\mathrm{SNR}_{1}, \mathrm{SNR}_{2}\right)$ that gives a point above the curve means that it is possible to achieve a rate $R$ for those $\left(\mathrm{SNR}_{1}, \mathrm{SNR}_{2}, \mathrm{SNR}_{12}, \mathrm{SNR}_{21}\right)$. A curve reaching the abscissa axis (resp., ordinate axis) means that receiver 2 (resp., 1) does not even need to receive information from its main channel, and that it will be able to retrieve the message only through the side information sent by the other receiver. It is clear from Fig. 1 that the $2 \mathrm{RC}$ scheme outperforms the non-interactive $\mathrm{CF}$ and DF schemes. The area between the 2RC bound and the minimum of the non-interactive $\mathrm{CF}$ and DF bounds represent all the main channel gains that support a reliable transmission only for the $2 \mathrm{RC}$ scheme. It shows that the $2 \mathrm{RC}$ scheme surpasses a simple selection scheme between the non-interactive $\mathrm{CF}$ and DF schemes.

Note that we obtain an orthogonal channel if, 1) we split $\left.\mathcal{Y}_{k}=\mathcal{Y}_{k}^{\mathrm{m}} \times \mathcal{Y}_{k}^{\mathrm{c}}, 2\right)$ we split $Y_{1}=\left(Y_{1}^{\mathrm{m}}, Y_{1}^{\mathrm{c}}\right), Y_{2}=\left(Y_{2}^{\mathrm{m}}, Y_{2}^{\mathrm{c}}\right)$ with $Y_{k}^{\mathrm{m}} \in \mathcal{Y}_{k}^{\mathrm{m}}, Y_{k}^{\mathrm{c}} \in \mathcal{Y}_{k}^{\mathrm{c}}, k=1,2$, and 3) we have

$$
p\left(y_{1}, y_{2} \mid x, x_{1}, x_{2}\right)=p\left(y_{1}^{\mathrm{m}}, y_{2}^{\mathrm{m}} \mid x\right) p\left(y_{1}^{\mathrm{c}} \mid x_{2}\right) p\left(y_{2}^{\mathrm{c}} \mid x_{1}\right),
$$

i.e., the received signals from the main channel are independent of the received signals from the cooperation links. The information-theoretic bounds derived under those general classes of channels can be specialized for any stationary memoryless channels compliant to the corresponding requirements. We decided to derive the bounds for the SISO Gaussian MC (Gaussian inputs and noises) as a special case of the class 


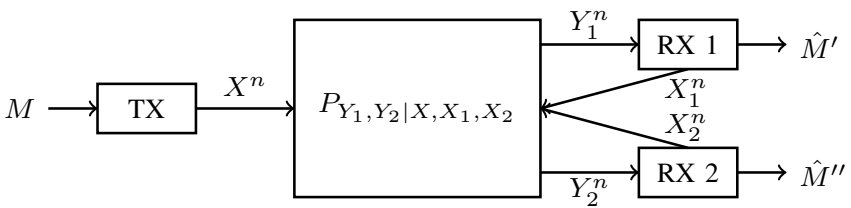

Fig. 2: The MC with non-orthogonal receiver cooperation.

of orthogonal stationary memoryless channels. The received signals at an instant $i$ are written as

$$
y_{1}^{\mathrm{m}}[i]=h_{1} x[i]+z_{1}[i] \text { and } y_{2}^{\mathrm{m}}[i]=h_{2} x[i]+z_{2}[i]
$$

where the transmitted signals are subject to the average power constraint $\sum_{i=1}^{n}|x[i]|^{2} \leq n P$, and the additive white Gaussian noises $(\mathrm{AWGN}) z_{k}[i] \sim \mathcal{C N}\left(0, \sigma^{2}\right)$ are independent across resources and receivers by assumption. The signal-to-noise ratios of the main channel are $\mathrm{SNR}_{k}=\left|h_{k}\right|^{2} \frac{P}{\sigma^{2}}, k=$ 1,2 , and the orthogonal noiseless finite capacity of the cooperation link from receiver $l$ to receiver $k$ is $C_{l k} \triangleq$ $\max _{p\left(x_{l}\right)} I\left(X_{l} ; Y_{k}^{\mathrm{c}}\right), k \neq l \in\{1,2\}$.

\section{STUDY OF THE 2RC SCHEME}

In this section, we study the $2 \mathrm{RC}$ scheme proposed in [14, Prop. 1] in the cases of 1) full-duplex cooperation links, 2) resource-sharing cooperation links, and 3) half-duplex cooperation links. Since we restrict our study to the bi-directional orthogonal cooperation links, we drop this identification in the remainder of the paper. In a nutshell, the $2 \mathrm{RC}$ scheme works as follows. In the first round, receiver $k$ uses $\mathrm{CF}$ to help receiver $l$ decode, and the second round, receiver $l$ uses DF to help receiver $k$ decode.

\section{A. Full-duplex cooperation}

From [14, Prop. 1], we obtain the following rate when the cooperation links are orthogonal to the main channel as in (1).

Proposition 1 (Full-duplex cooperation). The 2RC scheme achieves the following lower bound in a MC with full-duplex cooperation

$$
\begin{array}{r}
R_{2 R C}^{\text {ortho } \triangleq} \max _{k \neq l} \max _{\substack{o \\
k \leftrightarrow l}} \min \left\{I\left(X ; Y_{k}^{m}\right)+C_{l k}, I\left(X ; Y_{l}^{m}, \hat{Y}_{k}\right),\right. \\
\left.I\left(X ; Y_{l}^{m}\right)-I\left(Y_{k}^{m} ; \hat{Y}_{k} \mid X, Y_{l}^{m}\right)+C_{k l}\right\},
\end{array}
$$

where $\mathcal{P}_{k \leftrightarrow l}^{o}$ is the set of distributions $p(x) p\left(\hat{y}_{k} \mid y_{k}^{m}\right)$ with $\left|\hat{\mathcal{Y}}_{k}\right| \leq\left|\mathcal{Y}_{k}^{m}\right|+1$.

Corollary 1 (Gaussian channel with full-duplex cooperation). The 2RC scheme achieves the following lower bound expressed explicitly from Prop. 1 in a Gaussian MC with full-duplex cooperation

$$
\begin{aligned}
R_{2 R C}^{\text {Gauss } F D} \triangleq \max _{k \neq l} \min \left\{\log \left(1+\mathrm{SNR}_{k}\right)+C_{l k},\right. \\
\left.\log \left(1+\mathrm{SNR}_{l}+\frac{\mathrm{SNR}_{k}}{1+\Delta_{k}^{*}\left(C_{k l}\right)}\right)\right\},
\end{aligned}
$$

where $\Delta_{k}^{*}\left(C_{k l}\right) \triangleq \frac{1+\mathrm{SNR}_{l}+\mathrm{SNR}_{k}}{\left(1+\mathrm{SNR}_{l}\right)\left(2^{C} k l-1\right)}$.
Proof. We apply the Gaussian MC as defined in (2) to (3). The random variable $\hat{Y}_{k}=Y_{k}+N_{k}$ with $N_{k} \sim \mathcal{C N}\left(0, \Delta_{k}\right), k=$ 1,2 , represent the compression noise. Then, we set the second and third terms on the right hand side of (3) to be equal in their counterpart of Coro. 1 by tuning $\Delta_{k} \geq 0$ since the second term is a nonincreasing function of $\Delta_{k}$ and the third term is a nondecreasing function of $\Delta_{k}$ that intersect only once. This implies that the minimum of the two terms is maximized when the two terms are equal for the optimal value $\Delta_{k}^{*}\left(C_{k l}\right)$.

Note that in this case $C_{k l}$ and $C_{l k}$ are free parameters tunable for the cooperation links, and that there exist an optimal $\Delta_{k}^{*}\left(C_{k l}\right)$. Such a cooperation is suited for a network in which the cooperation links between the receivers are fixed, e.g., in practice each direction of cooperation between the devices could have its own link of fixed capacity.

\section{B. Resource-sharing cooperation}

Those achievable bounds can be specialized to a resourcesharing case between the cooperation links. Assume a resource (e.g., band) of normalized resource-sharing factor (e.g., bandwidth) $0 \leq w \leq 1$ that parametrizes the capacity of the cooperation links so that $C_{l k}=C w$, i.e., the link from receiver $l$ to $k$ is used over a ratio $w$ of the resource, and $C_{k l}=C(1-w)$, i.e., the link from receiver $k$ to $l$ is used over a ratio $(1-w)$ of the resource. From Coro 1 , we obtain the following rate with resource-sharing cooperation.

Corollary 2 (Gaussian channel with resource-sharing cooperation). The 2RC scheme achieves the following lower bound expressed explicitly from Coro. 1 in a Gaussian MC with resource-sharing cooperation

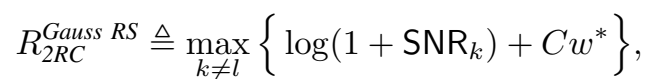

where $w^{*}$ is given in (6).

Proof. We introduce the resource-sharing factor $w$ in (4). Then, we set the first and second terms on the right hand side of (4) to be equal in their counterpart of Coro. 2 by tuning $0 \leq w \leq 1$ and taking into account the different intersections of the two terms as a function of $w$. This implies that the minimum of the two terms is maximized for $w^{*}$.

Note that $\Delta_{k}^{*}(C, w)$ is not present anymore in (5), but is given for completeness as $\Delta_{k}^{*}(C, w) \triangleq \frac{1+\mathrm{SNR}_{l}+\mathrm{SNR}_{k}}{\left(1+\mathrm{SNR}_{l}\right)\left(2^{C(1-w)}-1\right)}$. In this case $C$ and $w$ are free parameters tunable for the cooperation links, and there exist an optimal $\Delta_{k}^{*}(C, w)$ and $w^{*}$. Such a cooperation is suited for a network in which the cooperation links between the receivers can vary, e.g., in practice a link could exist between two devices, and the capacity of this link at disposal could vary between the use of the cooperation scheme.

\section{Half-duplex cooperation}

The 2RC scheme can be specialized to a half-duplex case between the multicast and the cooperation links. Assume a resource of normalized resource-sharing factors $0 \leq v, w \leq 1$ 


$$
w^{*}=\left\{\begin{array}{l}
0 \text { if } \log \left(1+\mathrm{SNR}_{k}\right)>\log \left(1+\mathrm{SNR}_{l}+\frac{\mathrm{SNR}_{k}}{1+\frac{1+\mathrm{SNR}_{l}+\mathrm{SNR}_{k}}{\left(1+\mathrm{SNR}_{l}\right)\left(2^{C}-1\right)}}\right) \\
1 \text { if } \log \left(1+\mathrm{SNR}_{k}\right)+C<\log \left(1+\mathrm{SNR}_{l}\right) \\
\frac{\log \left(\frac{\sqrt{\left({ }^{C} C\left(1+\mathrm{SNR}_{l}\right)\left(1+\mathrm{SNR}_{k}\right)\right)^{2}+4\left(2^{C}\right)\left(1+\mathrm{SNR}_{l}\right)\left(1+\mathrm{SNR}_{k}\right)\left(\mathrm{SNR}_{k}\right)\left(1+\mathrm{SNR}_{l}+\mathrm{SNR}_{k}\right)}-{ }_{2}^{C}\left(1+\mathrm{SNR}_{l}\right)\left(1+\mathrm{SNR}_{k}\right)}{2 \mathrm{SNR}_{k}\left(1+\mathrm{SNR}_{k}\right)}\right)}{C} \text { otherwise. }
\end{array}\right.
$$

that parametrizes the use of the multicast and of the capacity of the cooperation links so that the multicast is used over a ratio $v$ of the resource, $C_{l k, Q}=C_{Q}(1-v) w$, i.e., the link from receiver $l$ to $k$ is used over a ratio $(1-v) w$ of the resource, and $C_{k l, Q}=C_{Q}(1-v)(1-w)$, i.e., the link from receiver $k$ to $l$ is used over a ratio $(1-v)(1-w)$ of the resource. The bound presented in [14, Prop. 1] can be improved as underlined in the aforementioned paper by introducing a time-sharing random variable [16] $Q$, bounded as $|\mathcal{Q}| \leq 8$, and considering $|\mathcal{Q}|=3$. It can then be specialized to the orthogonal case as defined in (1), by setting $C_{l k, Q} \triangleq \max _{p\left(x_{l} \mid q\right)} I\left(X_{l} ; Y_{k}^{\mathrm{c}} \mid Q\right), k \neq l \in$ $\{1,2\}$, for this step.

Proposition 2 (Full-duplex cooperation with a time-sharing random variable). The $2 R C$ scheme achieves the following lower bound in a MC with full-duplex cooperation and a timesharing random variable

$$
\begin{aligned}
& R_{2 R C}^{\text {ortho }} T S \triangleq \\
& \max _{k \neq l} \max _{\mathcal{P}_{k \leftrightarrow l}^{o}(q)} \min \left\{I\left(X ; Y_{k}^{m} \mid Q\right)+C_{l k, Q}, I\left(X ; Y_{l}^{m}, \hat{Y}_{k} \mid Q\right),\right. \\
& \left.\quad I\left(X ; Y_{l}^{m} \mid Q\right)-I\left(Y_{k}^{m} ; \hat{Y}_{k} \mid X, Y_{l}^{m}, Q\right)+C_{k l, Q}\right\}, \quad
\end{aligned}
$$

where $\mathcal{P}_{k \leftrightarrow l}^{o}(q)$ is the set of distributions $p(q) p(x \mid q) p\left(\hat{y}_{k} \mid y_{k}^{m}, q\right)$ with $\left|\hat{\mathcal{Y}}_{k}\right| \leq\left|\mathcal{Y}_{k}^{m}\right|+1$ and $|\mathcal{Q}| \leq 8$.

We set $p(x) p\left(\hat{y}_{k} \mid y_{k}^{\mathrm{m}}\right)$ for $q=0, p\left(x_{l}\right)$ for $q=1$, and $p\left(x_{k}\right)$ for $q=2$. For $0 \leq v, w \leq 1$, the distribution of $Q$ is as follows, $p(q=0)=v, p(q=1)=(1-v) w$, and $p(q=2)=$ $(1-v)(1-w)$. Then, applying (2) on (7) gives

$$
\begin{aligned}
& R_{2 \mathrm{RC}}^{\mathrm{Gauss} \mathrm{HD}}=\max _{0 \leq v, w \leq 1} \\
& \min \left\{v \log \left(1+\mathrm{SNR}_{k}\right)+C_{Q}(1-v) w,\right. \\
& v \log \left(1+\mathrm{SNR}_{l}+\frac{\mathrm{SNR}_{k}}{1+\Delta_{k}}\right), \\
&\left.v \log \left(1+\mathrm{SNR}_{l}\right)+C_{Q}(1-v)(1-w)-v \log \left(1+\frac{1}{\Delta_{k}}\right)\right\} .
\end{aligned}
$$

The optimization of (8) is done in three steps, 1) on $\Delta_{k}$ for a fixed $v, w$ giving

$$
\Delta_{k}^{*}\left(C_{Q}, v, w\right) \triangleq \frac{1+\mathrm{SNR}_{l}+\mathrm{SNR}_{k}}{\left(1+\mathrm{SNR}_{l}\right)\left(2^{C_{Q} \frac{(1-v)(1-w)}{v}}-1\right)},
$$

2) on $w$ for $\Delta_{k}^{*}\left(C_{Q}, v, w\right)$ and a fixed $v$ giving $w^{*}$ in (10), and

3) on $v$ for $\Delta_{k}^{*}\left(C_{Q}, v, w\right)$ and $w^{*}$. Note that $v^{*}$ is not obvious in the general case because of the structure of $w^{*}$, but can be computed numerically in simulations, and thus we obtain the following lower bound.

Corollary 3 (Gaussian channel with half-duplex cooperation). The proposed scheme achieves the following lower bound expressed explicitly from Prop. 2 in a Gaussian MC with halfduplex cooperation

$R_{2 R C}^{\text {Gauss } H D} \triangleq \max _{k \neq l} \max _{0 \leq v \leq 1}\left\{v \log \left(1+\mathrm{SNR}_{k}\right)+C_{Q}(1-v) w^{*}\right\}$,

where $w^{*}$ is given in (10).

Note that $\Delta_{k}^{*}\left(C_{Q}, v, w\right)$ is not present anymore in (11). In this case $C_{Q}, v$ and $w$ are free parameters tunable for the cooperation links, and there exist an optimal $\Delta_{k}^{*}\left(C_{Q}, v, w\right)$ and $w^{*}$. Such a cooperation is suited for a network in which the receivers cannot transmit and receive over the same resource, and where the cooperation links between the receivers can vary, e.g., in practice a link could exist between two devices each equipped with only one interface for both the MC and the cooperation (e.g., one antenna and a switch between cellular network processing and local network processing), and the capacity of this link at disposal could vary between the use of the cooperation scheme.

\section{NUMERICAL RESULTS}

We first focus on the SISO Gaussian MC as defined in (2), and evaluate through numerical simulations the achievable rate of the 2RC schemes given in Coro. 1, Coro. 2 and Coro. 3. In order to provide a fair comparison the respective parameters $\Delta_{k}, v$ and $w$ are optimized for each bound. We study the impact of the cooperation links on the throughput of the channel. We set

$$
\frac{C_{12}+C_{21}}{2}=C=C_{Q}=C_{\text {coop }},
$$

meaning that the capacity of cooperation of the full-duplex scheme is equal to twice the maximal capacity of cooperation available to the resource-sharing and half-duplex schemes, since the receivers can transmit and receive simultaneously in full-duplex. Moreover, we assume for simplicity that the capacity of the cooperation links is symmetric, i.e.,

$$
C_{12}=C_{21} \text {. }
$$

In Fig. 3, we fix the SNR of the main channel, and plot the throughput in terms of spectral efficiency (bit $/ \mathrm{s} / \mathrm{Hz}$ ) by 


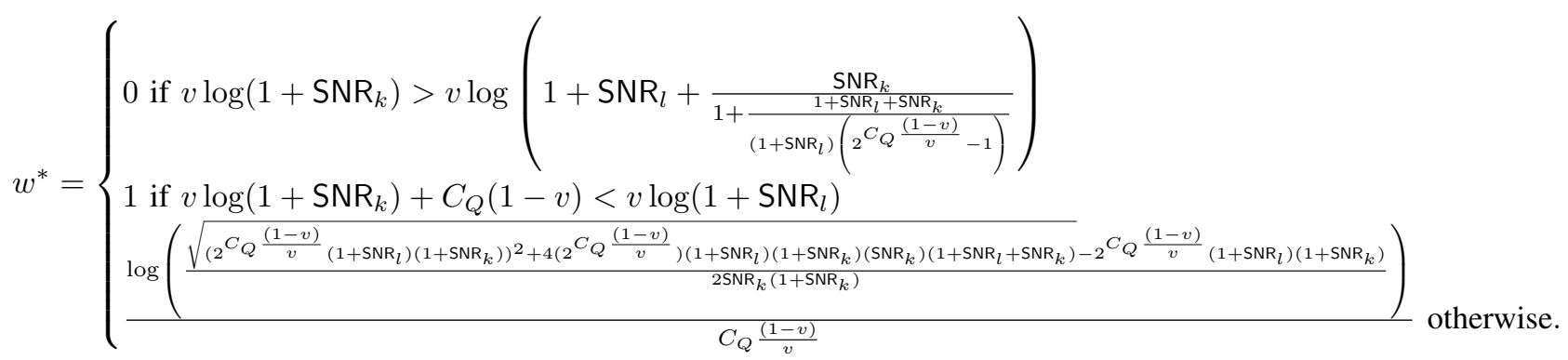

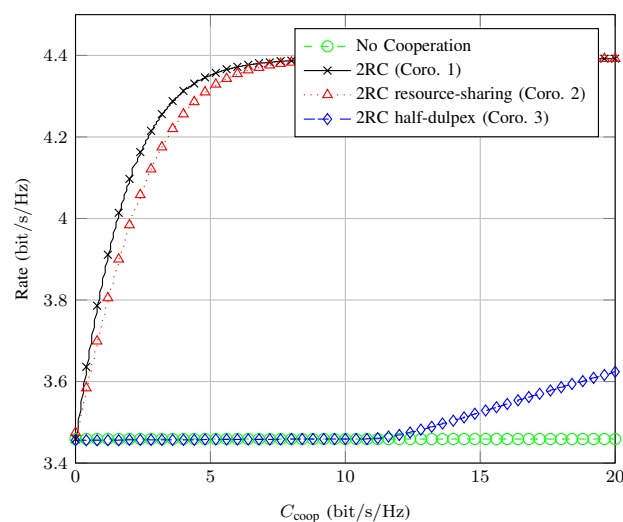

(a) $\mathrm{SNR}_{1}=\mathrm{SNR}_{2}=10 \mathrm{~dB}$ (symmetric).

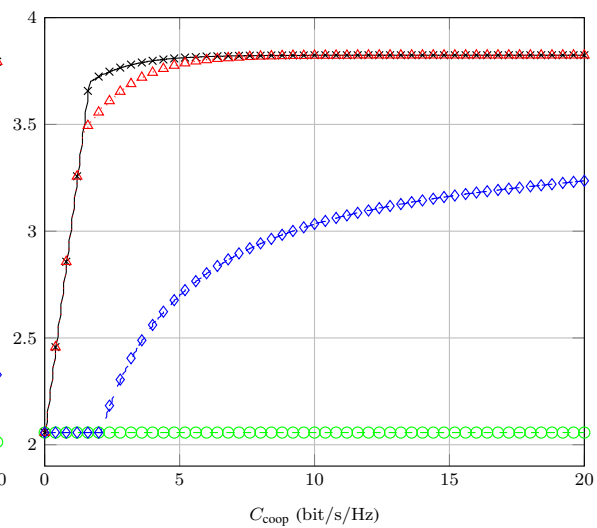

(b) $\mathrm{SNR}_{1}=5 \mathrm{~dB}, \mathrm{SNR}_{2}=10 \mathrm{~dB}$.

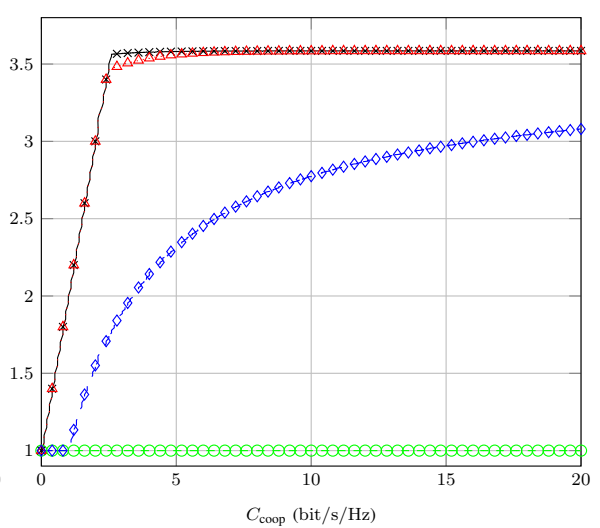

(c) $\mathrm{SNR}_{1}=0 \mathrm{~dB}, \mathrm{SNR}_{2}=10 \mathrm{~dB}$.

Fig. 3: Comparison of the bounds on the achievable rate for the Gaussian MC with receiver cooperation.

varying $C_{\text {coop }}$ from $0 \mathrm{bit} / \mathrm{s} / \mathrm{Hz}$ to $20 \mathrm{bit} / \mathrm{s} / \mathrm{Hz}$. In Fig. 3a, the main channel is symmetric with a SNR of $10 \mathrm{~dB}$ at each receiver, while in Fig. $3 b$ and Fig. $3 c$ the main channel is asymmetric. In all the configurations, the $2 \mathrm{RC}$ scheme and its special cases outperform the "no cooperation" scheme. When $C_{\text {coop }} \rightarrow 0$ the schemes tend to the "no cooperation" lower bound while when $C_{\text {coop }} \rightarrow \infty$ the schemes tend to the the broadcast bottleneck $\log \left(1+\mathrm{SNR}_{1}+\mathrm{SNR}_{2}\right)$. In Fig. 4, we represent the factors of the $2 \mathrm{RC}$ resource-sharing and halfduplex schemes corresponding to the setups of Fig. 3.

In Fig. 3b and Fig. 3c, the 2RC full-duplex and resourcesharing bounds increase linearly with the capacity of cooperation at low $C_{\text {coop }}$, while it increases non-linearly in the symmetric case. This behavior of the $2 \mathrm{RC}$ full-duplex cooperation scheme is due, as underlined in [14], to the fact that it achieves the same rate that the non-interactive CF scheme in the SISO case when the main channel is symmetric and the capacity of the cooperation links is symmetric as in (13). In other words, in Fig. 3a, since both receivers achieve the same performance the DF operation does not bring any gain (when one receiver can decode, the other one can also decode). In the asymmetric case, since one receiver has more information than the other, it uses DF toward the weakest receiver to increase the achievable rate of the common message, until the weakest receiver is not the one setting the rate anymore. This gives the linear increase of the bound at low $C_{\text {coop }}$, since the first term of (4) is active. At high $C_{\text {coop }}$, the second term of (4) is active. Then, the bound increases non-linearly and tends to the broadcast bottleneck due to the $\mathrm{CF}$ operation. This is the case since both receivers virtually achieve the same performance as a result of the DF cooperation which can be fully performed for this range of $C_{\text {coop }}$. As for the $2 \mathrm{RC}$ resource-sharing scheme, we can see that when $w^{*}=1$ in Fig. $4 \mathrm{~b}$ and Fig. $4 \mathrm{c}$ the bound increases linearly respectively in Fig. $3 \mathrm{~b}$ and Fig. 3c. Substituting $w^{*}=1$ into (5) explains the linear increase of the bound.

In Fig. 3 the 2RC half-duplex bounds are equal to the "no cooperation" bounds at low $C_{\text {coop. }}$. We can see that when $v^{*}=1$ in Fig. 4 the multicasting is preferred over the cooperation. Substituting $v^{*}=1$ into (11) explains the behavior of the bound. The behavior of the $2 \mathrm{RC}$ half-duplex bounds at high $C_{\text {coop }}$ can be analyzed in the same manner as the ones presented for $2 \mathrm{RC}$ full-duplex and resource-sharing bounds.

We learn from Fig. 4 that the DF/CF ratio (represented by the $w^{*}$ ) is higher at low $C_{\text {coop }}$ than at high $C_{\text {coop }}$, meaning that the DF is preferred when the cooperation links are weak and that the $\mathrm{CF}$ is preferred when the cooperation links are strong. This is due to the fact that at low $C_{\text {coop }}$ the system improves the rate of the weakest user (the one receiving the DF from the strongest user) which is the limiting rate, while at high $C_{\text {coop }}$ the system improves the description compressed by the 


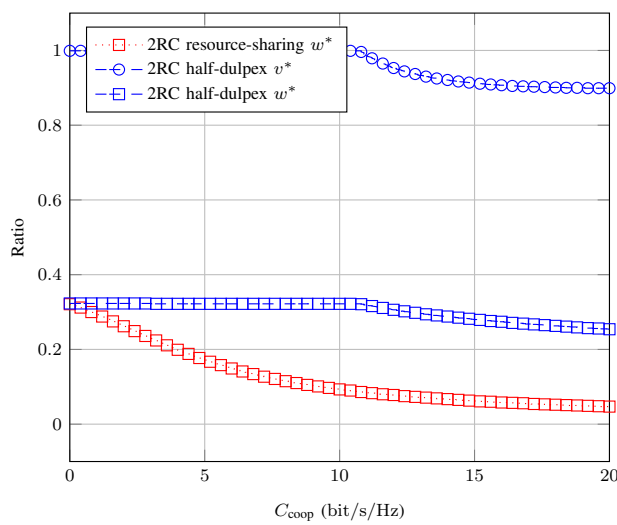

(a) $\mathrm{SNR}_{1}=\mathrm{SNR}_{2}=10 \mathrm{~dB}$ (symmetric).

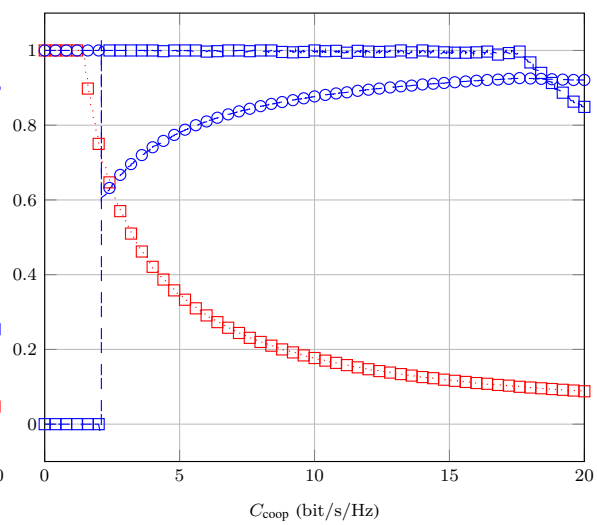

(b) $\mathrm{SNR}_{1}=5 \mathrm{~dB}, \mathrm{SNR}_{2}=10 \mathrm{~dB}$.

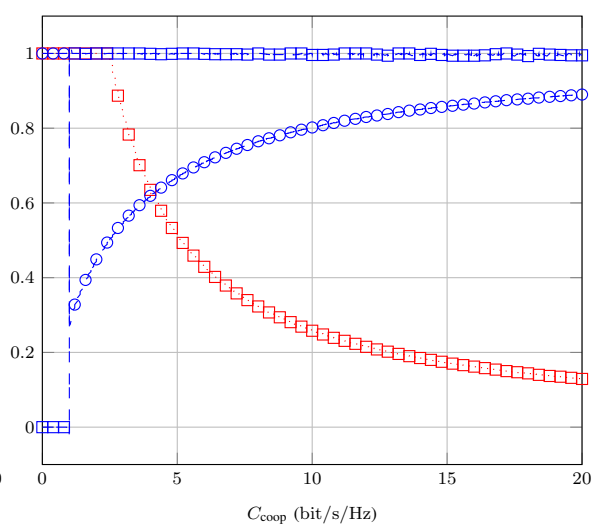

(c) $\mathrm{SNR}_{1}=0 \mathrm{~dB}, \mathrm{SNR}_{2}=10 \mathrm{~dB}$.

Fig. 4: Comparison of the factors of the $2 \mathrm{RC}$ resource-sharing and $2 \mathrm{RC}$ half-duplex schemes for the Gaussian MC with receiver cooperation.

weakest user toward the strongest user to tend to the broadcast bottleneck. We also learn that the $\mathrm{DF} / \mathrm{CF}$ ratio is higher when the main channel is asymmetric for the same reasons.

Note that the resource-sharing scheme has performances comparable to the ones of the full-duplex scheme even though (12) sets the maximal capacity of cooperation available to the resource-sharing to be equal to half the capacity of cooperation of the full-duplex. This comes from the DF/CF ratio. Moreover, the resource-sharing schemes takes into account more practical aspects than the full-duplex scheme regarding the complexity of implementation. Thus, a suboptimal equivalent of the resource-sharing scheme would ensure good performance in practice while remaining at low complexity. We point out the fact that at high $C_{\text {coop }}$, the achievable rate is virtually not impacted by the $\mathrm{DF} / \mathrm{CF}$ ratio since it is limited by the broadcast bottleneck. Finally, while a suboptimal equivalent of the half-duplex scheme would lead to the easiest implementation, its performances would be very low.

Such a study would lead to similar conclusions in the non-orthogonal case by defining similar sub-cases to the ones presented herein. However, due to the non-linearity of the bounds in this latter case, the explanation regarding the resource-sharing factors would mainly be obtained through numerical results and not closed-form expressions.

\section{CONCLUSION}

In this paper, we investigated the impact of receiver cooperation and of the factors that can tune this cooperation on the throughput of a two-receiver MC. The scheme used for the study is the $2 \mathrm{RC}$ scheme, which is a scheme that provably outperforms existing schemes in which no interaction is exploited. The numerical results showed that the optimal DF/CF ratio decreases as the cooperation links get stronger, and that it also decreases as the main channel becomes symmetric. The optimal DF/CF ratio is important mainly when the cooperation links are weak, since when the cooperation links are strong the achievable rate is limited by the broadcast bottleneck. Finally, the resource-sharing scheme has performances comparable to the ones of the full-duplex scheme for a much lower complexity of implementation, while the half-duplex presents very low performances.

\section{REFERENCES}

[1] T. Cover, "Broadcast channels," IEEE Trans. Inf. Theory, vol. 18, no. 1 pp. 2-14, Jan. 1972

[2] P. P. Bergmans, "Random coding theorem for broadcast channels with degraded components," IEEE Trans. Inf. Theory, vol. 19, no. 2, pp. 197 207, Mar. 1973.

[3] K. Marton, "A coding theorem for the discrete memoryless broadcast channel," IEEE Trans. Inf. Theory, vol. 25, no. 3, pp. 306-311, May 1979.

[4] E. van der Meulen, "Three-terminal communication channels," $A d v$. Appl. Prob., vol. 3, no. 1, pp. 120-154, Spring 1971.

[5] T. Cover and A. El Gamal, "Capacity theorems for the relay channel," IEEE Trans. Inf. Theory, vol. 25, no. 5, pp. 572-584, Sep. 1979.

[6] G. Kramer, M. Gastpar, and P. Gupta, "Cooperative strategies and capacity theorems for relay networks," IEEE Trans. Inf. Theory, vol. 51, no. 9, pp. 3037-3063, Sep. 2005.

[7] S. H. Lim, Y.-H. Kim, A. El Gamal, and S.-Y. Chung, "Noisy network coding," in Proc. Inf. Theory Workshop (ITW), 2010, pp. 1-5.

[8] — "Noisy network coding," IEEE Trans. Inf. Theory, vol. 57, no. 5, pp. 3132-3152, May 2011.

[9] J. Hou and G. Kramer, "Short message noisy network coding with a decode-forward option," IEEE Trans. Inf. Theory, vol. 62, no. 1, pp. 89-107, Jan. 2016

[10] A. Behboodi and P. Piantanida, "Cooperative strategies for simultaneous and broadcast relay channels," IEEE Trans. Inf. Theory, vol. 59, no. 3 pp. 1417-1443, Mar. 2013.

[11] R. Dabora and S. D. Servetto, "Broadcast channels with cooperating decoders," IEEE Trans. Inf. Theory, vol. 52, no. 12, pp. 5438-5454, Dec. 2006.

[12] Y. Liang and V. V. Veeravalli, "Cooperative relay broadcast channels," IEEE Trans. Inf. Theory, vol. 53, no. 3, pp. 900-928, Mar. 2007.

[13] Y. Liang and G. Kramer, "Rate regions for relay broadcast channels," IEEE Trans. Inf. Theory, vol. 53, no. 10, pp. 3517-3535, Oct. 2007.

[14] V. Exposito, S. Yang, and N. Gresset, "A two-round interactive receiver cooperation scheme for multicast channels," in Proc. 54th Allerton Conf. on Commun., Control, and Comput., 2016, to appear.

[15] T. Cover and J. Thomas, Elements of Information Theory, Second Edition. Wiley-Interscience, 2006.

[16] A. El Gamal and Y.-H. Kim, Network Information Theory. Cambridge Univ. Press, 2012. 